\title{
Efficient electronic passivation scheme for computing low-symmetry compound semiconductor surfaces in density-functional theory slab calculations
}

\author{
Su-Hyun Yoo ๑, ${ }^{*}$ Liverios Lymperakis $\odot$, and Jörg Neugebauer \\ Department of Computational Materials Design, Max-Planck-Institut für Eisenforschung GmbH, \\ Max-Planck-Straße 1, D-40237 Düsseldorf, Germany
}

(Received 24 February 2021; accepted 1 April 2021; published 16 April 2021)

\begin{abstract}
Removing artificial bands from the back side of surface slabs with pseudohydrogen atoms has become the method of choice to boost the convergence of density-functional theory (DFT) surface calculation with respect to slab thickness. In this paper we apply this approach to semipolar compound semiconductor surfaces, which have recently become attractive for device applications. We show that approaches employing saturation of dangling bonds by pseudohydrogen atoms alone are inadequate to properly passivate the surfaces, remove spurious surface states from the fundamental band gap, and achieve flat band conditions in the slab. We propose and successfully apply to technologically interesting semipolar wurtzite surfaces of III-N, III-V, and II-VI semiconductors a reconstruction-inspired passivation scheme that utilizes native anions to passivate cation dangling bonds and pseudohydrogen atoms to obey the electron counting rule and compensate for polarization-induced surface-bound charges. This scheme is generic and robust and can be straightforwardly implemented in DFT investigations of low-symmetry surfaces as well as in high-throughput and machine learning studies.
\end{abstract}

DOI: 10.1103/PhysRevMaterials.5.044605

\section{INTRODUCTION}

Surfaces are a key component in modern materials and devices. They control epitaxial growth as well as the properties and efficiency of materials used in electronic, optoelectronic, sensing, or catalysis applications [1-3]. For instance, the electronic structure of surfaces, i.e., density and energy levels of the surface states, has been proposed to control the properties of a two-dimensional electron gas (2DEG) in high-electronmobility transistors (HEMTs) [4,5]. The vast majority of modern optoelectronic and/or power electronic devices based on III-nitrides or $\mathrm{ZnO}$ employ the low-index $\{0001\}$ surfaces of the wurtzite (WZ) crystal (see Ref. [6] and references therein). However, these surfaces and interfaces are accompanied by spontaneous polarization fields which are detrimental to the efficiency of the optoelectronic devices due to the quantum confined Stark effect [7]. Semipolar planes, i.e., (hkil) planes with a nonzero $h, k$, or $i$ and a nonzero $l$ index, have smaller polarization fields and provide an alternative to the polar growth direction [8-12]. Semipolar planes also constitute the side facets of detrimental defects at surfaces or interfaces such as the V-pits [13] or of nanostructures such as quantum dots (QDs) [14].

\footnotetext{
*yoo@mpie.de
}

Published by the American Physical Society under the terms of the Creative Commons Attribution 4.0 International license. Further distribution of this work must maintain attribution to the author(s) and the published article's title, journal citation, and DOI. Open access publication funded by the Max Planck Society.
A prerequisite to control the growth and properties of surfaces is to achieve an atomic-scale understanding of their structural, thermodynamic, and electronic properties. First-principles-based computational approaches constitute nowadays a workhorse to study materials [15]. A common approach to model surfaces in density-functional theory (DFT) calculations is to employ the repeated slab geometry with periodic boundary conditions $[16,17]$. In this approach, the bulk transitional symmetry in the direction perpendicular to the surface is broken by inserting a sufficiently thick vacuum between consecutive thin films, which leave a typical thickness of a few monolayers (MLs).

To guarantee converged surface properties using the slab approach, it is essential to minimize errors arising from the finite-size slab and the long-range interactions between the two bounding surfaces. In the case of a symmetric slab where the two surfaces are identical, these interactions over the slab can be minimized by increasing the slab thickness at the expense of higher computational cost. However, in the case of an asymmetric slab bounded by two symmetry-inequivalent surfaces, additional treatments are required. Firstly, the different surface dipoles and work functions of the two sides of the slab result in an artificial electric field over the vacuum region. Furthermore, intragap surface states may pin the Fermi level at both sides of the slab and result in a spurious electron transfer from high-energy states at the one surface to lower-energy states at the other surface, inducing an electric field over the slab region. The former challenge is commonly addressed by employing the dipole correction scheme [18]. The latter challenge is tackled by following the principles of the electron counting rule (ECR) [19] and saturating the surface dangling bonds (dbs) with hydrogen or pseudohydrogen atoms (psHs) [20]. In materials with two or more symmetry-inequivalent 
polar directions, such as wurtzite semiconductors, the study of the technologically important polar surfaces is further complicated by spontaneous polarization along the surface normal. This causes a net surface charge and a macroscopic electric field over the slab [21,22]. Recently, this has been addressed by modifying the valency of the psHs to compensate for the polarization-induced surface-bound charges [22].

The above-mentioned schemes have been successfully applied to study both nonpolar and polar semiconductor surfaces (see, e.g., Refs. [22] and [23]). However, in this paper, we show that these schemes fail to efficiently passivate $\mathrm{WZ}$ semipolar surfaces. Even when passivating these surfaces following the ECR, and compensating for the polarizationinduced bound charges, deep intragap surface states remain. These gap states introduce an artificial electric field in the slab region, and they (i) result in a poor convergence with respect to slab thickness and (ii) prevent direct calculations of the surface electronic structure. In the following we identify the origin of the aforementioned challenge. Based on these insights we propose a generic scheme to efficiently passivate semipolar planes. The paper is organized as follows. In Sec. II the methodology is presented, and the available passivation schemes are presented and discussed in Sec. III. In Sec. IV we discuss the failure of the commonly applied passivation schemes for surfaces with doubly coordinated cations and identify the origin of this failure. Building on this insight, we propose a reconstruction-inspired passivation scheme. The performance and robustness of this approach are demonstrated for selected semipolar planes and compound semiconductors. Section V summarizes the key findings.

\section{METHODOLOGY}

All DFT calculations are performed using the Vienna $a b$ initio simulation package (VASP) [24,25], employing the projector augmented wave (PAW) approach [26]. The Perdew-Burke-Ernzerhof generalized gradient approximation (GGA-PBE) is used for the exchange-correlation approximation [27,28]. The Ga $3 d$ electrons are treated as valence states. For the plane-wave basis set, the kinetic energy cutoff is set to $500 \mathrm{eV}$. A $\Gamma$-centered $8 \times 8 \times 6 k$-point grid is used for WZ bulk systems, to perform Brillouin-zone integrations. $8 \times 3 \times 1$ and $4 \times 4 \times 1$ grids are used for the $1 \times 1$ surface unit cell of the $\{1 \overline{1} 02\}$ and $\{11 \overline{2} 2\}$ slabs, respectively.

Slabs of WZ GaN, AlN, ZnO, and GaAs are considered. The calculated lattice constants ( $a$ and $c / a$ ), internal parameters $(u)$, and direct band-gap energies $\left(E_{\mathrm{g}}\right)$ are in agreement with experimental and previous theoretical works (see Table S1 in the Supplemental Material [29]). The spontaneous polarization constants with respect to the reference phase of zinc blende $(\mathrm{ZB})\left(\mathbf{P}^{\mathrm{WZ}-\mathrm{ZB}}\right)$ are listed in Table $\mathrm{S} 2$ in the Supplemental Material [29] and are in agreement with previous calculations.

Stoichiometric slabs are used to model the semipolar surfaces. To construct the surfaces, the bulk is cleaved in a way to minimize the number of dbs. The $\{1 \overline{1} 02\}$ and $\{11 \overline{2} 2\}$ slabs consist of 31 and 32 cation-anion dimers, respectively. The thicknesses of $\mathrm{GaN}, \mathrm{AlN}, \mathrm{ZnO}$, and GaAs slabs are 31.23, $30.24,31.85$, and $38.07 \AA$ for the $\{1 \overline{1} 02\}$ slabs and 21.74 , $21.05,22.11$, and $27.22 \AA$ for the $\{11 \overline{2} 2\}$ slabs, respectively.
Consecutive slabs are separated by a vacuum with a thickness of $18 \AA$. The electronic relaxation is carried out until the total energy changes less than $10^{-5} \mathrm{eV}$. Only psHs are allowed to relax. Relaxation is performed until the energy change in consecutive steps is less than $10^{-4} \mathrm{eV}$ per surface unit cell. All other atoms are kept fixed at their bulklike positions. The dipole correction scheme [18] is employed to decouple different surface dipoles in asymmetric slabs through the vacuum.

\section{SURFACE PASSIVATION SCHEMES IN DFT CALCULATIONS}

The repeated slab geometry is the standard approach in DFT calculations to model surfaces. In this geometry a slab is bound by two free surfaces. For many technologically important surface orientations these are symmetry inequivalent. Prominent examples are the $\{0001\}$ polar surfaces in III-nitrides or $\mathrm{ZnO}$. A common way to create surfaces is to cut along a plane that minimizes the number of dbs after cleavage. In the case of polar WZ surfaces this results in a slab bound by the (0001) surface with one cation $\mathrm{db}$ and the $(000 \overline{1})$ surface with one anion $\mathrm{db}$ per $1 \times 1$ unit cell. For these half-truncated surfaces, both dbs are partially filled with the cation db state lying higher in energy than the anion db state. Hence a spurious charge transfer occurs from the (0001) to the (0001) side of the slab. Even for slabs bounded by symmetry-equivalent surfaces, such as the nonpolar (1100) and (1120) surfaces, due to the finite slab thickness db states from the two sides of the slab will interact. This interaction lifts the degeneracy of the upper and lower surface states giving rise to a spurious splitting of these states.

To circumvent the above issues, the dbs at the "bottom" side of the slab, i.e., the surface which is not under investigation, have to be passivated. The passivation is commonly done by fictitious hydrogen atoms with fractional electrons and protons, so-called psHs, that comply with the ECR [19]. For instance, assuming that $\mathrm{N}(\mathrm{Ga})$ atoms in the $\mathrm{WZ}$ crystal donate 5/4 (3/4) electrons to each bond, the dbs at these atoms are passivated with psHs with valency of $0.75(1.25)$ [denoted hereafter as $\mathrm{psH}_{0.75}\left(\mathrm{psH}_{1.25}\right)$ ].

The aforementioned scheme (denoted henceforth as the conventional passivation scheme) has been successfully applied on many semiconductor surfaces. However, as recently shown [22], applying the conventional scheme to polar surfaces, on systems exhibiting spontaneous polarization, (i) causes a poor size convergence with respect to the slab thickness and (ii) fails to unambiguously describe the surface band structure. The origin of these issues is the failure of the conventional passivation scheme to realize a charge-neutral surface [22]. Rather, spontaneous polarization causes a net surface charge which induces a macroscopic electric field over the slab.

The presence of an electric field results in canted band edges. Once the slab exceeds a critical thickness, even an overlap between the valence band maximum (VBM) on the one side and the conduction band minimum (CBM) on the other side, referred to as breakdown, occurs. As a result the effective band gap of the semiconductor becomes zero. This (i) results in a poor size convergence, (ii) induces errors in the calculated surface energies, and (iii) artificially shifts bulk 
and/or surface states into the band gap. The latter prevents achievement of a proper alignment with the bulk band edges. These challenges have been successfully addressed by modifying the valency of the psHs to account both for the ECR and for the spontaneous-polarization-induced surface-bound charges according to the surface theorem of the modern theory of polarization [30]. For example, in the case of $\mathrm{ZnO}(000 \overline{1})$ surfaces, the $\mathrm{O}$ dbs have to be passivated by $\mathrm{psH}$ with 0.48 valency instead of 0.5 as in the conventional scheme.

The aforementioned passivation scheme has been successfully tested and applied on polar WZ slabs [22]. In the following we will apply it to semipolar surfaces. A prerequisite to apply the suggested scheme to semipolar surfaces is to identify the spontaneous-polarization-induced surface-bound charges $\sigma_{\text {semipolar }}$. These can be written as

$$
\sigma_{\text {semipolar }}=\frac{A_{\{0001\}}}{A_{\text {semipolar }}} \mathbf{P}^{\mathrm{WZ}-\mathrm{ZB}} \cos (\Theta),
$$

where $\mathbf{P}^{\mathrm{WZ}-\mathrm{ZB}}$ is the spontaneous polarization constant of the WZ phase with respect to the $\mathrm{ZB}$ one, $\Theta$ is the angle between the polar axis and the surface normal, and $A_{\{0001\}}$ and $A_{\text {semipolar }}$ are the surface areas of the polar and semipolar surface unit cells, respectively. The angle-dependent spontaneous polarizations $\mathbf{P}^{\mathrm{WZ}-\mathrm{ZB}} \cos (\Theta)$ for four materials in the $\mathrm{WZ}$ phase, i.e., GaN, AlN, $\mathrm{ZnO}$, and GaAs, are shown in Fig. S1 in the Supplemental Material [29].

Once the polarization-induced surface-bound charges have been identified, they are evenly distributed over the psHs, i.e., the polarization charge has to be divided by the number of dbs $\left(N_{\mathrm{db}}\right)$. The valency of the psHs $\left(Z_{\mathrm{psH}^{*}}\right)$ is thus given by

$$
Z_{\mathrm{psH}^{*}}=\left(\frac{Z_{\mathrm{C} / \mathrm{A}}}{N_{\text {coord }}}+\frac{\sigma_{\text {semipolar }}}{N_{\mathrm{db}}}\right),
$$

where $Z_{\mathrm{C} / \mathrm{A}}$ is the valency of the cation or the anion and $N_{\text {coord }}$ stands for the coordination number. For example, the calculated surface-bound charge for the GaN $(\overline{1} 10 \overline{2})$ surface [left surface of the slab shown in Fig. 1(a)] is $-0.036 e$ per surface unit cell. The cleaved (1102) surface has three Ga $\mathrm{dbs}$ and one $\mathrm{N} \mathrm{db}$. Therefore the psHs passivating the $\mathrm{Ga}$ and $\mathrm{N}$ dbs have valencies of 1.241 [i.e., $(5 / 4)-(0.036 / 4)$ ] and of 0.741 [i.e., $(3 / 4)-(0.036 / 4)]$, respectively. In the same way, the $\mathrm{Ga}$ and $\mathrm{N}$ dbs at the (1102) semipolar surface bounding the other side of the slab are passivated by $\mathrm{psH}_{1.259}$ and $\mathrm{psH}_{0.759}$, respectively. The surface-bound charges for all semipolar surfaces of WZ phases studied in this paper are listed in Table S3 in the Supplemental Material [29].

\section{RESULTS AND DISCUSSION}

\section{A. Failure of passivation schemes at surfaces with low-coordinated cations}

Let us first examine how the conventional passivation scheme works for semipolar surfaces of WZ materials. As was discussed in our recent work [22], due to the failure of this scheme to reproduce the semi-infinite bulk limit, the presence of an electric field in the semipolar slab is expected. The magnitude of this field should equal the polarization-induced field, i.e., $\mathcal{E}=\sigma / \varepsilon$, where $\mathcal{E}$ and $\varepsilon$ are the electric field and (a)
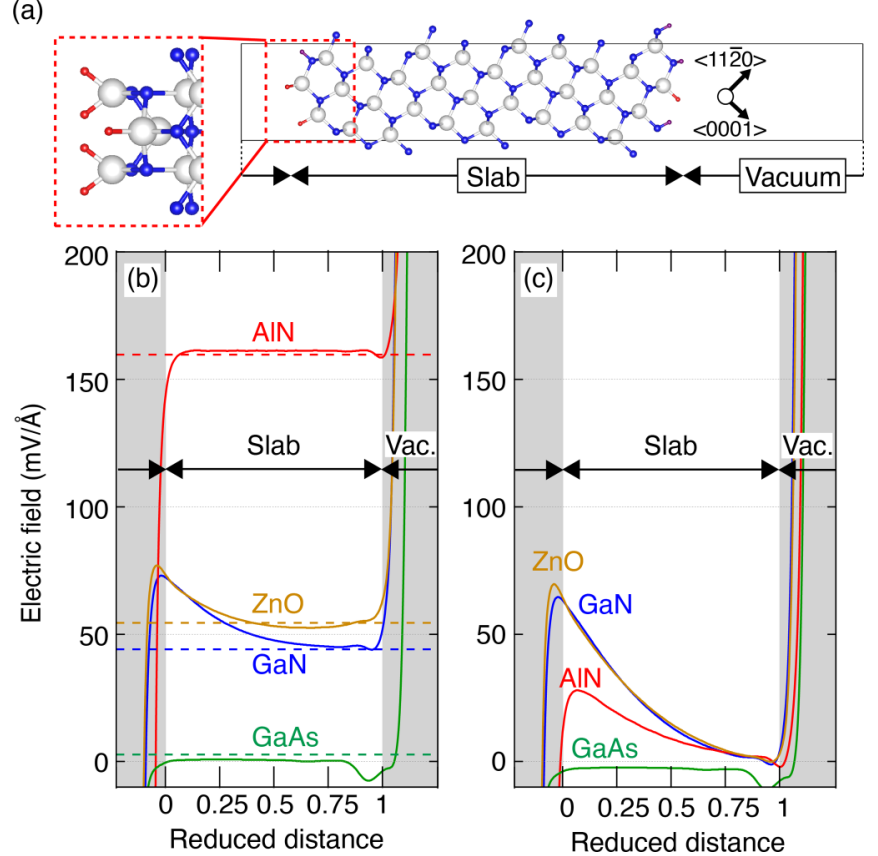

FIG. 1. (a) Schematic representation of the atomic structures of the WZ $\{1 \overline{1} 02\}$ slab in side view along [1100]. Cations and anions are depicted by white and blue balls, respectively. The psHs saturating cation and anion dbs are shown as red and purple balls, respectively.

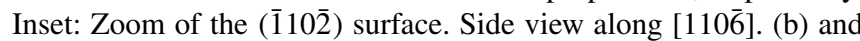
(c) Calculated electric field of four WZ $\{1 \overline{1} 02\}$ slabs: GaN (blue), $\mathrm{AlN}$ (red), $\mathrm{ZnO}$ (orange), and GaAs (green), with different passivation schemes $\{$ (b) conventional scheme and (c) modified psH scheme [22] \}plotted as a function of the normalized distance along the surface normal in units of slab thickness. The gray shaded areas denote the vacuum (Vac.) region. For the sake of clarity the vacuum region is not shown. The electrostatic potential profiles in both the slab and the vacuum regions are shown in Fig. S2 in the Supplemental Material [29].

the high-frequency dielectric constant along the semipolar direction, respectively.

In Fig. 1(b), the calculated electric field is plotted normal to the $\{1 \overline{1} 02\}$ surface. In all cases, i.e., for $\mathrm{GaN}, \mathrm{AlN}, \mathrm{ZnO}$, and GaAs, a finite electric field is present in the slab. Its magnitude at the right part of the slab, i.e., at the (1102) surface, coincides as expected with the field induced by spontaneous polarization (horizontal dashed lines). However, at the left part of slab, i.e., at the $(\overline{1} 10 \overline{2})$ surface, a strong bending of the electric field for $\mathrm{GaN}$ and $\mathrm{ZnO}$ occurs. Interestingly, AlN does not show such a bending, and the magnitude of the electric field is constant inside the slab. For GaAs, the electric field in the slab is almost two orders of magnitude smaller than in the other materials. This can be traced back to the roughly two orders of magnitude smaller spontaneous polarization constant of WZ-GaAs (see Table S2 in the Supplemental Material [29]).

The qualitative differences between $\mathrm{GaN}$ and $\mathrm{ZnO}$ on the one side and AlN on the other indicate that different mechanisms contribute to the electric field in these slabs. One mechanism is the spontaneous polarization, which is not compensated by the conventional passivation scheme [22]. We therefore eliminate it by modifying the valency of the psHs. 

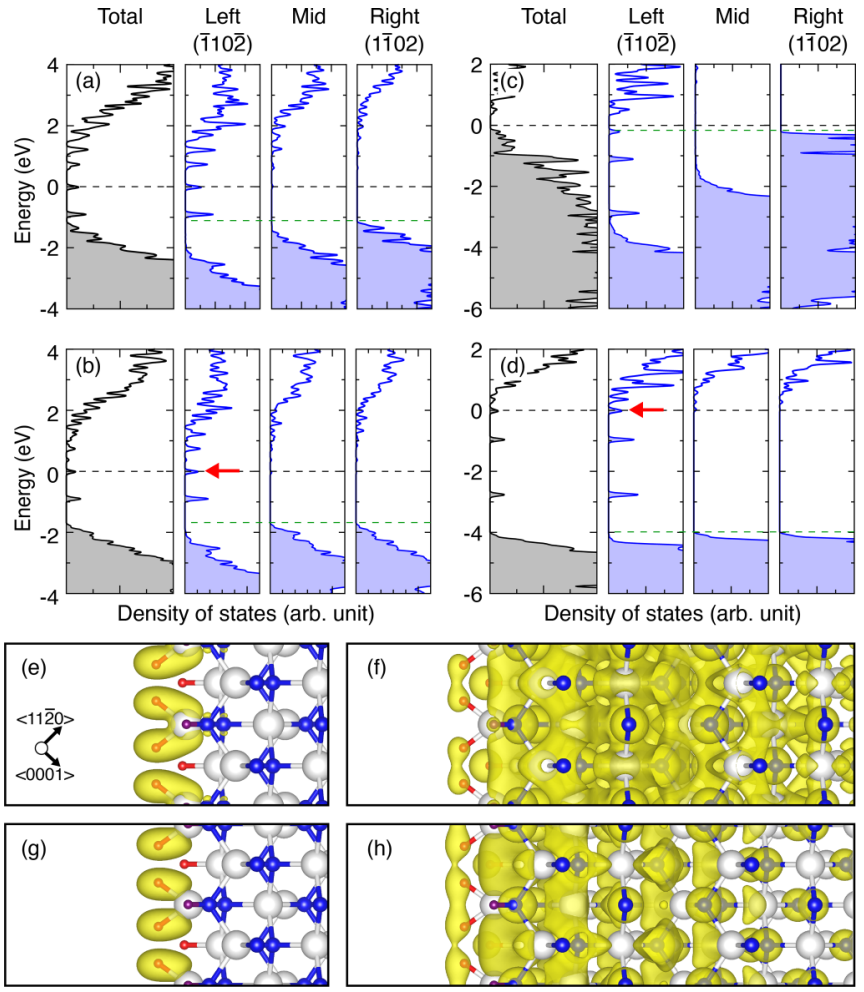

FIG. 2. Total and layer-resolved density of states at the left, middle (Mid), and right side of the $\{1 \overline{1} 02\}$ slab for (a) and (b) GaN and (c) and (d) AlN. In (a) and (c) the conventional passivation scheme has been applied, and in (b) and (d) the free surfaces are passivated by modified psHs. Shaded areas denote occupied states. The horizontal black dashed lines indicate the positions of the highest occupied states. The horizontal green dashed lines indicate the position of the highest occupied state at the right side of the slab and are drawn as a guide to the eye to highlight the bending of the valence band edge. In all cases, the highest occupied state is set to zero. Partial charge densities of the peaks denoted by the red arrows in (b) and (d) are shown in (e) and (f) for GaN and (g) and (h) for AlN, respectively. Each of these peaks consists of two states energetically lying very close at the $\Gamma$ point. One of these states originates from the metallic interactions between cations [(f) and (h)] with high dispersion and the the other originates from the psH-cation bonds [(e) and $(\mathrm{g})]$ with low dispersion in a large part of the Brillouin zone (see Fig. S3 in the Supplemental Material [29]).

These pseudoatoms are specifically constructed to compensate for the polarization-induced surface-bound charges. The calculated electric fields are plotted in Fig. 1(c). As can be seen, GaN, AlN, and $\mathrm{ZnO}$ show qualitatively the same behavior: At the (1102) surface of the slab the magnitude of the electric field is now negligible, as expected in the absence of polarization. However, at the other side of the slab a strong upward bending is observed. We can therefore conclude that spontaneous polarization is not the origin of the spurious bending of the electric field at the $(\overline{1} 10 \overline{2})$ surface.

To understand its origin, we plot in Figs. 2(a)-2(d) the total and layer-resolved density of states (LDOS) of GaN [Figs. 2(a) and 2(b)] and AIN [Figs. 2(c) and 2(d)] with the conventional passivation scheme [Figs. 2(a) and 2(c)] and with modified psHs [Figs. 2(b) and 2(d)]. The corresponding density of states (DOS) of $\mathrm{ZnO}$ and GaAs slabs is shown in Figs. S4 and S5 in the Supplemental Material [29].

Although both surfaces of the slabs are passivated, the surface becomes metallic in all considered cases: As shown in Fig. 2 the Fermi level is pinned at partially occupied states. In the case of the conventional passivation scheme the origin behind metallicity could be a Fermi level pinning at the CBM or a high-lying state at one side of the slab and at the VBM or a low-lying state at the other side. Indeed, this is the case for the AlN slab [see Fig. 2(c)]: The Fermi level is pinned at the VBM (right surface) and a high-lying surface state at the left side of the slab. Although AlN is a wide-band-gap material (calculated band gap $4.06 \mathrm{eV}$ ), the large spontaneous polarization together with the relatively thick slab (30.24 $\AA$ ) causes a breakdown. This explanation appears to be in contrast to the fact that $\mathrm{GaN}$ has a smaller band gap (calculated band gap $1.74 \mathrm{eV}$ ). However, since the magnitude of the spontaneous polarization of $\mathrm{GaN}$ is considerably smaller than that of AlN, the Fermi level is not pinned at the VBM [see Fig. 2(a)], resulting in an insulating surface. At the passivated (left) surface, however, the Fermi level is pinned at a surface state. The presence of such a surface state clearly shows that the conventional passivation scheme fails. Even if the dbs are passivated with modified psHs [see Figs. 2(b) and 2(d)] the db states are not completely removed. The fact that the valence band edge in the region between the middle and the right side of the slab is flat shows that the modified psHs correctly remove the polarization field [see Figs. 2(b) and 2(d)].

In order to examine the origin of the Fermi level pinning, which is a clear indication that the passivation at the $(\overline{1} 10 \overline{2})$ surface fails, we inspect the partially occupied surface states at the GaN and AlN slabs. The partial charge density of these states is plotted in Figs. 2(e)-2(h). As can be seen, these states arise predominantly from metallic interactions between neighboring surface metal atoms [see Figs. 2(f) and 2(h) for GaN and AlN, respectively] as well as from bonding states between psHs and the doubly coordinated surface metal atoms [see Fig. 2(e) for GaN and Fig. 2(g) for AlN]. If the passivation scheme works as intended, the metal-psH bonding states should be fully occupied and should lie deep in the valence band. However, due to imperfect passivation, metallic states between neighboring cations in the surface layer move into the gap and pin the Fermi level. These states lie close to the bottom of the bulk conduction band. As a consequence the metal-psH states strongly hybridize with the conduction band. Due to the strong hybridization the $\mathrm{psH}$-induced states extend deep into the slab. The large vertical extent is clearly demonstrated by the partial charge density shown in Figs. 2(f) and 2(h) and Fig. S6 in the Supplemental Material [29]. Since the hybridized state is metallic, the metal-psH bond charge is equally distributed over the hybridized state, resulting in strong polarization. This polarization builds up a strong surface dipole, which manifests the strong bending of the electric field at the (imperfectly) passivated side of the slab.

\section{B. Origin of the partially occupied states at the passivated surface}

The failure of psHs to effectively passivate $\mathrm{dbs}$ and remove surface induced deep states from the band gap is only 
observed at cleaved $\mathrm{GaN}$, AlN, and $\mathrm{ZnO}$ semipolar planes when doubly coordinated cation atoms are present. Passivation works well for (i) semipolar planes with doubly coordinated anions, (ii) WZ GaAs semipolar planes, and (iii) polar WZ surfaces, i.e., $\{0001\}$ surfaces.

It is well known that III-nitride surfaces exhibit a unique stabilization mechanism: Under molecular beam epitaxy (MBE) and metal-rich conditions, excess metal adlayers are stabilized on the surface [31-33]. The stabilization of a metallic structure over an ECR structure has been explained by (i) the short interatomic distance of the metal atoms in the cation sublattice and (ii) the extreme binding energy of $\mathrm{N}_{2}$ molecules which compete against the formation of $\mathrm{Ga}-\mathrm{N}$ bonds [34]. The cation interatomic distances in $\mathrm{GaN}, \mathrm{AlN}$, and $\mathrm{ZnO}$ are only $\approx 8-18 \%$ larger than those in the corresponding metal bulk phases, i.e., orthorhombic $\alpha$-Ga, cubic fcc-Al, and hexagonal hcp-Zn (see Table S4 in the Supplemental Material [29]). Metallic interactions may emerge even between fully coordinated cations, though in highly strained regions in the neighborhood of edge-type dislocation cores [35]. In contrast, GaAs has an $\approx 20 \%$ larger lattice constant than $\mathrm{GaN}$ rendering the interatomic distance of group III atoms almost 50\% larger than in bulk $\alpha-\mathrm{Ga}$. Hence, owing to the larger lattice constant of GaAs, low-coordinated Ga atoms at WZ GaAs semipolar planes are too far away to form metallic bonds.

Figures 2(f) and 2(h) show that the intragap states are related to bonds between neighboring psHs. These bonds occur when Ga atoms with doubly broken dbs are passivated with psHs. The formation of these extra bonds violates the assumptions behind ECR: According to these, psH should be monovalent and form only a single bond to the metal atom.

To verify the hypothesis of incomplete passivation by the formation of unintended bonds, we consider the bonding and antibonding states of a molecule consisting of two psHs with valency of 1.25 . To test the formation of undesired bonds, we calculate the energy levels of these states as a function of the interatomic distance [see Fig. 3(a)]. Even though the calculated equilibrium bond length is $\approx 0.8 \AA$, we find strong interactions. These interactions lift the degeneracy of the low-lying orbitals, denoted as $\sigma_{\mathrm{s}}$ and $\sigma_{\mathrm{s}}^{*}$ in Fig. 3(a). These interactions exist even at a rather large interatomic distance of $1.5 \AA$, i.e., distances that are considerably larger than the $\mathrm{H}-\mathrm{H}$ distance in $\mathrm{H}_{2}$ molecule. Considering the fact that the distance between psHs at the ( $1 \overline{1} 02)$ GaN surface is $\approx 1.4 \AA$, unintended bonds between these psHs form. As pointed out above, these extra bonds are not captured by the ECR. As a consequence a proper saturation of $\mathrm{Ga}$ dbs by psHs is not achieved.

A potential strategy to address the aforementioned issue is to manually reduce the psH-Ga distances at the (11)02) surface. By reducing this distance to $0.8 \AA$ the interatomic distance between the psHs becomes larger than $1.6 \AA$. In this case, the surface states are removed from the band gap, and a state with high density at the valence band (VB) edge emerges as indicated by red arrows in Fig. 3(c). Reducing the distance even further, an extreme case is reached where the distance becomes zero, i.e., a "fusion" of the psHs with the surface $\mathrm{Ga}$ atoms occurs. In this extreme scenario, partially filled states are induced again in the band gap [see Fig. 3(d)]. These observations imply that the spurious partially filled states are
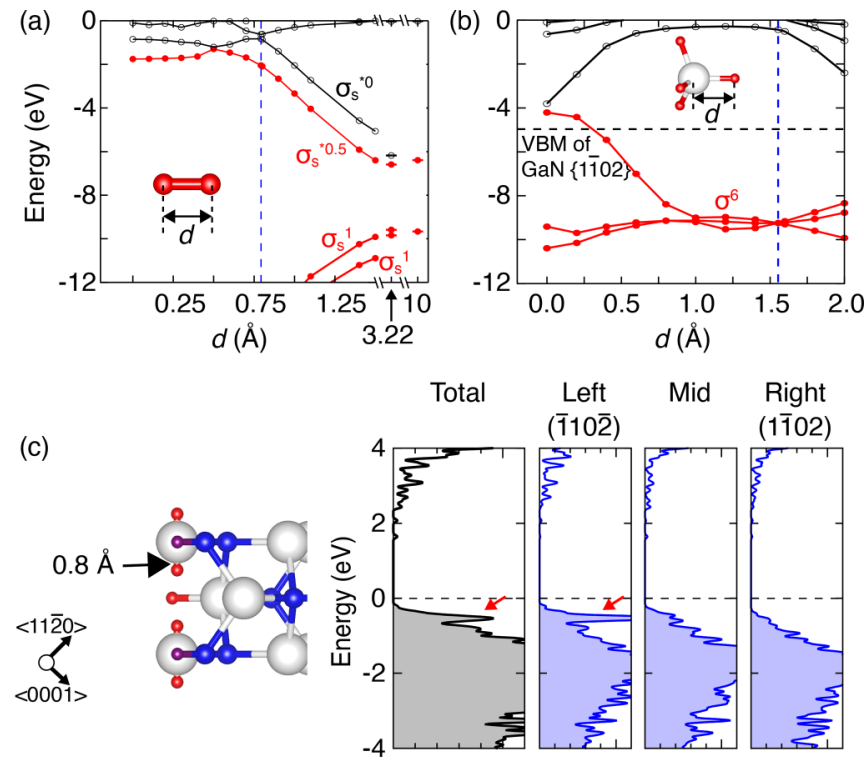

(d)
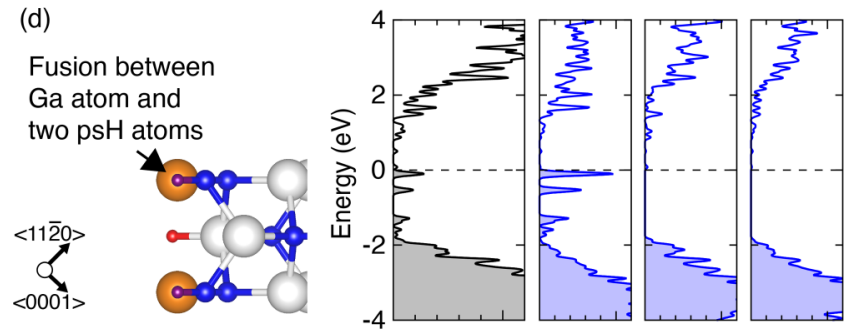

Density of states (arb. unit)

FIG. 3. Calculated Kohn-Sham molecular orbital energies. The energies are given with respect to the vacuum level (i.e., $0 \mathrm{eV}$ ). Energies for (a) the $\left(\mathrm{psH}_{1.25}\right)_{2}$ and (b) the $\mathrm{Ga}\left(\mathrm{psH}_{1.25}\right)_{4}$ molecules as a function of bond distance $d$. In $\mathrm{Ga}\left(\mathrm{psH}_{1.25}\right)_{4}$, two bonds between the $\mathrm{Ga}$ atom and $\mathrm{psH}_{1.25}$ are manipulated. The other two bonds are fixed at the equilibrium distance. The occupied or partially occupied states and the empty states are represented by solid and open circles, respectively. In (a) the vertical dashed line represents the equilibrium distance of the $\left(\mathrm{psH}_{1.25}\right)_{2}$ molecule. In (b) the equilibrium bond distance and the VBM of the $\{1 \overline{1} 02\}$ slab are indicated by the vertical blue and the horizontal dashed lines, respectively. Insets: Schematic representation of the $\left(\mathrm{psH}_{1.25}\right)_{2}$ and $\mathrm{Ga}\left(\mathrm{psH}_{1.25}\right)_{4}$ molecules, respectively. Left panels of (c) and (d): Ball and stick model of the (110 $\overline{2})$ surface. Cations and anions are depicted by large white and small blue balls, and psHs passivating cation and anion dbs are depicted by smaller red and purple balls, respectively. In (c) the $\mathrm{psH}_{1.241}-\mathrm{Ga}$ distances are adjusted to $0.8 \AA$. In (d) the $\mathrm{psH}_{1.241}$ atoms are placed on top of the surface Ga atoms. The "fused" atoms are denoted with the large orange balls. Right panels of (c) and (d): Total and layer-resolved DOS of the slabs shown in the left panels.

strongly affected by the Ga-psH and psH-psH interatomic distances: As the Ga-psH distance is reduced both the metallic and $\mathrm{psH}$ interactions are decoupled, and the passivation of the $\mathrm{Ga}$ dbs removes the intragap states. A disadvantage of this approach is that the Ga-psH bonding states rise in energy as the interatomic distance between them decreases. Eventually, these states enter the band gap. This behavior is illustrated in Fig. 3(b), where the molecular orbital energies of a cluster consisting of a $\mathrm{Ga}$ atom and four $\mathrm{psH}_{1.25}$ atoms at tetrahedral 

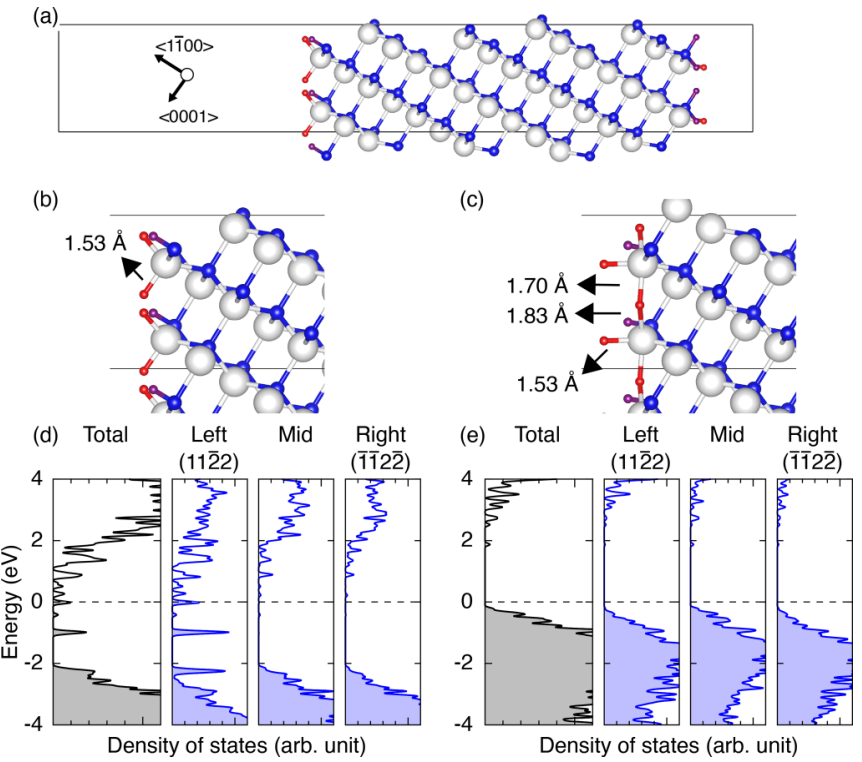

FIG. 4. (a) Ball and stick model of the GaN $\{11 \overline{2} 2\}$ slab (side view). Cations and anions are depicted by large white and small blue balls, respectively. psHs passivating cation and anion dbs are depicted by smaller red and purple balls, respectively. The rectangle indicates the supercell. (b) and (c) show a zoom of the (112̄2) surface. In (b) the Ga-psH distances are adjusted to $1.53 \AA$, and in (c) their positions are optimized by atomic relaxations. (d) and (e) show the total and layer-resolved DOS of the $\{11 \overline{2} 2\}$ slab. The (112̄2) side is passivated as shown in (b) and (c), respectively.

positions are plotted as a function of the $\mathrm{Ga}-\mathrm{psH}_{1.25}$ distance. As the distance is reduced, the bonding and antibonding states shift upward and downward, respectively. At interatomic distances shorter than $\approx 0.4 \AA$ these states lie above the valence band edge of the $\{1 \overline{1} 02\}$ GaN slab.

Let us now focus on another technologically relevant semipolar surface. Specifically, we consider the GaN (11222) surface. This surface has again doubly coordinated cations [see Fig. 4(a)]. When their dbs are passivated by psHs placed at the low-coordinated cations and using the same equilibrium distances that were needed to passivate the (1102) surface, deep states appear in the band gap [see Fig. 4(d)]. Thus the same mechanism that hinders proper passivation of the (1102) surface is also active here. Interestingly, if relaxation of the psHs is allowed, the surface states are removed from the band gap [see Figs. 4(c) and 4(e)]. Similarly, surface states are not observed on the $\mathrm{ZnO}$ and AlN (112̄2) surfaces (see Figs. S7 and S8 in the Supplemental Material [29]). The relaxed geometry undergoes a phase transition [Fig. 4(c)] where the originally twofold coordinated surface cations form each three bonds with the surrounding psHs. This phase resembles a pseudohydride. In the pseudohydride the shortest interatomic distance between any two psHs is larger than $\approx 2 \AA$. Interaction (bond formation) is thus effectively suppressed. The pseudohydride is semiconducting with the bonding and antibonding states lying deep in the valence and conduction bands of the slab, respectively. Therefore it efficiently passivates the (11) 2 ) surface.

The aforementioned cases indicate that the efficient passivation of semipolar planes is case specific and can be achieved by different physical mechanisms: The $\{1 \overline{1} 02\}$ slabs require manual and careful adjustment of the cation-psH distances, while efficient passivation of the (112 2$)$ surface can be achieved by the spontaneous formation of a pseudohydride phase. However, a computationally convenient passivation scheme should be generic, simple, and based on a small number of physical principles or mechanisms. We address this issue in the following section.

\section{Reconstruction-inspired passivation scheme}

A common mechanism that governs surface reconstructions of compound semiconductors to obey the ECR is to alter the stoichiometry of the cleaved surface. The polar $(0001)$ GaN surface constitutes a characteristic example: The $2 \times 2$ $\mathrm{N}$ adatom reconstruction, which is favorable energetically under N-rich conditions in molecular beam epitaxy, obeys the ECR. Its stoichiometry deviates from the cleaved surface by placing a $\mathrm{N}$ atom on a hollow site every $2 \times 2$ surface cells. Motivated by this behavior, we propose a passivation scheme for semipolar WZ surfaces that we name the reconstructioninspired passivation scheme (RIPS). The key idea underlying this scheme is that passivation of doubly broken cation dbs by psHs alone is not adequate to achieve charge neutrality and flat band conditions. We therefore propose a two-step approach. In the first step all doubly broken cation dbs are either passivated by native anion atoms or removed leaving only anions with doubly broken dbs. In the second step, psHs with a valency that obeys the ECR and compensates for the polarization-induced surface-bound charges are placed on the remaining dbs. This approach is general and independent of the orientation of the surface. Furthermore, to effectively passivate dbs, no manual adjustment of the positions of the psHs is needed. Rather, only a routine atomic relaxation has to be applied. Therefore the proposed approach is robust and minimizes the necessary user effort. It is thus also well suited for high-throughput surface calculations and/or machine learning studies.

To illustrate the application of the proposed scheme, we consider the GaN ( $\overline{1} 10 \overline{2})$ surface. As shown in Fig. 5(a) the unit cell of the initially cleaved surface consists of a triply and a doubly coordinated Ga atom plus a triply coordinated $\mathrm{N}$ atom. Following the RIPS approach, a $\mathrm{N}$ atom is adsorbed at the bridgelike position between two neighboring doubly coordinated $\mathrm{Ga}$ atoms. The position of the adsorbed $\mathrm{N}$ atom is optimized by atomic relaxation keeping all other atoms fixed. The new surface structure consists of a triply coordinated cation and a doubly and a triply coordinated anion. In the next step the dbs of the low-coordinated atoms are passivated with psHs. To obey ECR and to remove the field induced by spontaneous polarization, the valency of $\mathrm{psH}$ is 1.241 for passivating $\mathrm{Ga}$ dbs and 0.741 for $\mathrm{N}$ dbs [see Fig. 5(b)]. The position of the psHs is relaxed by keeping all other atoms fixed.

The thus constructed structure is free of any doubly coordinated $\mathrm{Ga}$ atoms. Therefore metal-metal interactions (bonds) are expected to be eliminated. The interatomic distance of the doubly coordinated $\mathrm{N}$ atoms is found to be identical to the lattice constant of $\mathrm{GaN}(\approx 3.2 \AA)$. Hence the $\mathrm{N}-\mathrm{N}$ separation is considerably larger than the equilibrium bond length of 
(a)

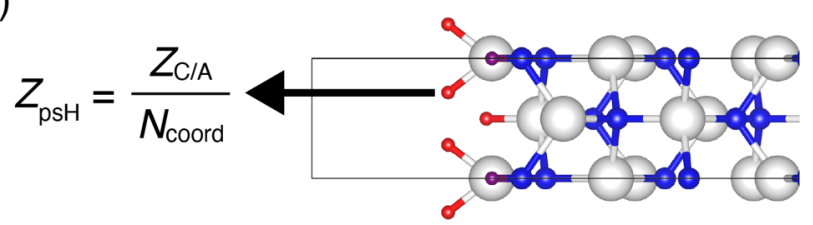

(b)

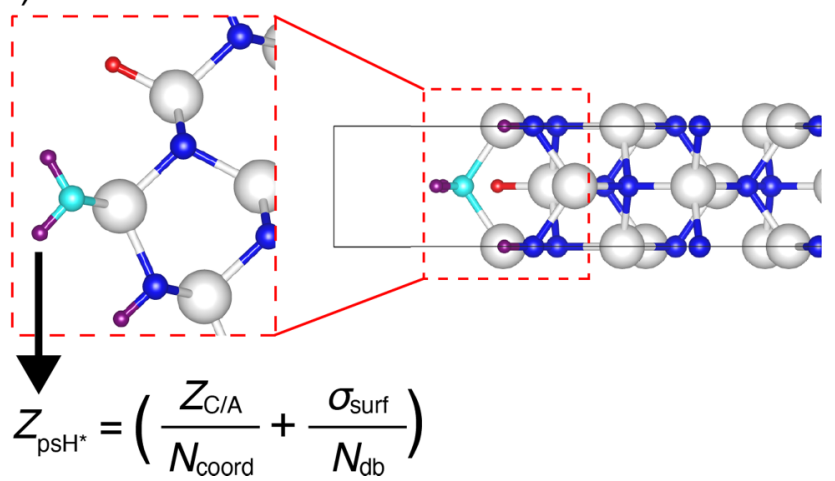

FIG. 5. Schematic illustration of (a) the passivation scheme based on psHs only and (b) RIPS. Cations and anions are depicted by white and blue balls, respectively. The psHs saturating cation and anion dbs are presented by the red and purple balls, respectively. The additional native anion employed to passivate the doubly coordinated cation is shown as a small cyan ball. The valency of the psHs (i.e., $Z_{\mathrm{psH}}$ and $\left.Z_{\mathrm{psH}^{*}}\right)$ is also given.

a $\mathrm{N}_{2}$ molecule $(\approx 1 \AA)$. Therefore also $\mathrm{N}-\mathrm{N}$ interactions are expected to be absent.

As revealed by the total DOS and LDOS shown in Fig. 6(c) the above formulated expectations are fulfilled: The deep and/or near band edge states induced by metallic interactions and/or interactions between psHs by the conventional passivation scheme are completely absent. Figure 6(a) demonstrates that RIPS also eliminates the polarization field in the
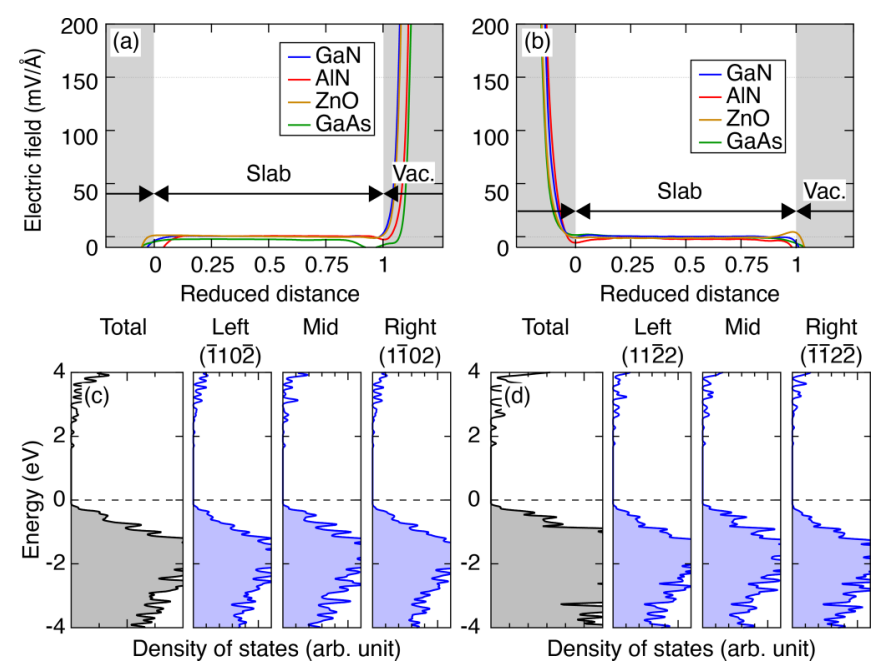

FIG. 6. Calculated electric fields of a WZ (a) $\{1 \overline{1} 02\}$ and (b) $\{11 \overline{2} 2\}$ slab. The field is plotted as a function of normalized distance along the surface normal. All surfaces are passivated with RIPS. The gray shaded areas denote the vacuum region. (c) and (d) show the total DOS and LDOS of $\{1 \overline{1} 02\}$ and $\{11 \overline{2} 2\}$ GaN slabs, respectively.
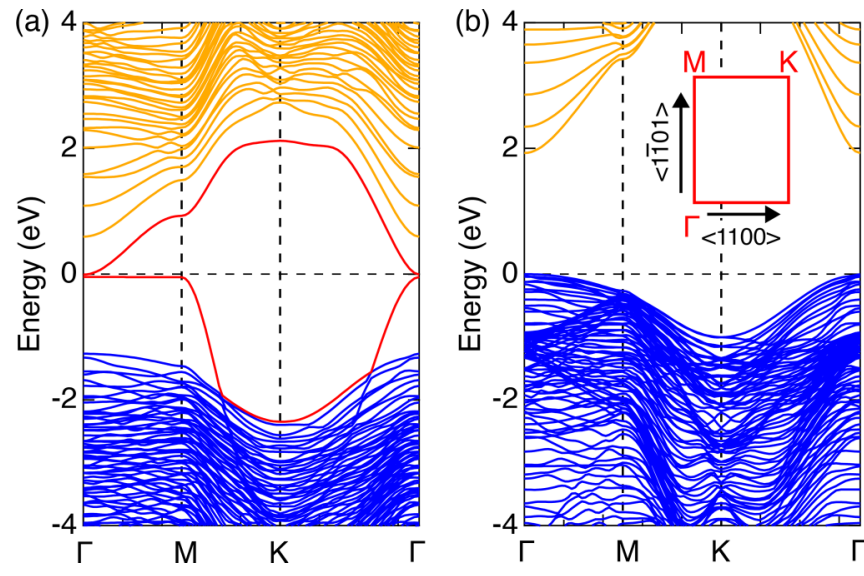

FIG. 7. Band structure of $\mathrm{GaN}\{1 \overline{1} 02\}$ slab where both surfaces are passivated by (a) the conventional passivation scheme and (b) RIPS. In (a) the red curves denote states arising from the $(\overline{1} 10 \overline{2})$ surface. Inset in (b): Schematic representation of the 2D Brillouin zone. In both (a) and (b) the highest occupied state is set to 0 .

slab and produces flat band conditions. Therefore both the polarization charges and the partially filled deep states induced by the conventional passivation scheme vanish.

The applicability of RIPS has also been tested for other semipolar GaN orientations [see Figs. 6(b) and 6(d)] as well as for other binary alloys (see Figs. S9 and S10 in the Supplemental Material [29]). Furthermore, in this paper we explicitly consider heteropolar semiconductors with cations having larger atomic radius than the anions. However, RIPS is more general and can be applied to binaries containing cations with smaller radius than the anions such as $\mathrm{BAs}, \mathrm{BSb}$, or $\mathrm{AlSb}$. In this case, all doubly broken anion dbs have to be either passivated by native cation atoms or removed leaving only cations with doubly broken dbs.

Having an approach that faithfully eliminates spurious surface states within the band gap constitutes a significant advancement in modeling semipolar and/or low-symmetry surfaces of III-V and II-VI semiconductors. As shown in Ref. [22], eliminating the spurious surface states considerably improves convergence with respect to slab thickness. Employing RIPS will thus allow one to use computational resources more efficiently and to study larger surface reconstructions and/or unit cells.

The flat band conditions realized by RIPS allow one also to extract the surface electronic band structures directly. This feature becomes specifically important in optoelectronic or electronic materials where the energetic position and the density of the surface states play a dominant role. Examples are the formation of space charge regions due to Fermi level pinning at surfaces or interfaces [36] or the computation of the properties of 2DEG in HEMTs [37]. The surface band structures shown in Figs. 7(a) and 7(b) demonstrate the excellent working of RIPS. Employing conventional passivation, the $\mathrm{GaN}\{1102\}$ surface slab is wrongly predicted to be metallic. As discussed before the origins are deep states induced by the spurious metallic interactions and interactions between neighboring psHs at the ( $(\overline{1} 10 \overline{2})$ surfaces [see Fig. 7(a)]. Due to the band bending induced by these states a proper alignment 
with the bulk band edges cannot be achieved. However, passivation of the $(\overline{1} 10 \overline{2})$ surface by RIPS cleans the band gap from spurious surface states and allows for a correct description of the surface electronic structure and alignment with the bulk band structure \{see Fig. 7(b) and Figs. S3 and S11 in the Supplemental Material [29]\}.

\section{CONCLUSIONS}

In conclusion, we demonstrate that the conventional passivation scheme employed in DFT slab calculations fails when applied to semipolar compound semiconductors' surfaces. Specifically, we show that it is unable to passivate surfaces containing cations with more than a single $\mathrm{db}$. In this case, unintended bands between neighboring cations and psHs introduce deep states into the fundamental band gap. The failure of complete passivation is a serious obstacle to study these technologically highly relevant surfaces. We therefore have developed an improved passivation scheme denoted as the "reconstruction-inspired passivation scheme" (RIPS). It consists of the following two steps: In the first step the surface stoichiometry is altered by adsorbing the minimum necessary number of native anions to passivate cation doubly broken dbs. In the second step, all surface dbs are passivated by psHs to (i) obey the ECR and (ii) compensate for the polarization-induced surface-bound charges. This scheme has been successfully applied to compute the energy and band structure of III-nitride, III-V, and II-VI semipolar surfaces. The scheme is general and easy to implement. It can be thus straightforwardly employed in DFT-based surface investigations as well as in high-throughput and/or machine learning studies.

\section{ACKNOWLEDGMENTS}

This project has received funding from the ECSEL Joint Undertaking (JU) project UltimateGaN under Grant Agreement No. 826392. The JU receives support from the European Union's Horizon 2020 research and innovation program and Austria, Belgium, Germany, Italy, Slovakia, Spain, Sweden, Norway, and Switzerland.
[1] L. J. Brillson, Surfaces and Interfaces of Electronic Materials (Wiley-VCH, Berlin, 2010).

[2] W. Mönch, Semiconductor Surfaces and Interfaces (Springer, Berlin, 2001).

[3] G. Somorjai, Surf. Sci. 89, 496 (1979).

[4] M. S. Miao, J. R. Weber, and C. G. Van de Walle, J. Appl. Phys. (Melville, NY) 107, 123713 (2010).

[5] M. Higashiwaki, S. Chowdhury, M.-S. Miao, B. L. Swenson, C. G. Van de Walle, and U. K. Mishra, J. Appl. Phys. (Melville, NY) 108, 063719 (2010).

[6] J. Zúniga-Pérez, V. Consonni, L. Lymperakis, X. Kong, A. Trampert, S. Fernández-Garrido, O. Brandt, H. Renevier, S. Keller, K. Hestroffer, M. R. Wagner, J. S. Reparaz, F. Akyol, S. Rajan, S. Rennesson, T. Palacios, and G. Feuillet, Appl. Phys. Rev. 3, 041303 (2016).

[7] P. Waltereit, O. Brandt, A. Trampert, H. T. Grahn, J. Menniger, M. Ramsteiner, M. Reiche, and K. H. Ploog, Nature (London) 406, 865 (2000).

[8] T. Wang, Semicond. Sci. Technol. 31, 093003 (2016).

[9] B. Corbett, Z. Quan, D. V. Dinh, G. Kozlowski, D. O’Mahony, M. Akhter, S. Schulz, P. Parbrook, P. Maaskant, M. Caliebe, M. Hocker, K. Thonke, F. Scholz, M. Pristovsek, Y. Han, C. J. Humphreys, F. Brunner, M. Weyers, T. M. Meyer, and L. Lymperakis, in Light-Emitting Diodes: Materials, Devices, and Applications for Solid State Lighting XX, edited by H. Jeon, L.-W. Tu, M. R. Krames, and M. Strassburg, Proceedings of the SPIE Vol. 9768 (International Society for Optics and Photonics, Bellingham, WA, 2016), pp. 163-171.

[10] A. E. Romanov, T. J. Baker, S. Nakamura, and J. S. Speck, J. Appl. Phys. (Melville, NY) 100, 023522 (2006).

[11] K. Xing, C. Tseng, L. Wang, P. Chi, J. Wang, P. Chen, and H. Liang, Appl. Phys. Lett. 114, 131105 (2019).

[12] T. Liu, J. Zhang, X. Su, J. Huang, J. Wang, and K. Xu, Sci. Rep. 6, 26040 (2016)
[13] S. Besendörfer, E. Meissner, A. Tajalli, M. Meneghini, J. A. Freitas, J. Derluyn, F. Medjdoub, G. Meneghesso, J. Friedrich, and T. Erlbacher, J. Appl. Phys. (Melville, NY) 127, 015701 (2020).

[14] B. Daudin, J. Phys. Condens. Matter 20, 473201 (2008).

[15] K. Lejaeghere, G. Bihlmayer, T. Björkman, P. Blaha, S. Blügel, V. Blum, D. Caliste, I. E. Castelli, S. J. Clark, A. Dal Corso, S. de Gironcoli, T. Deutsch, J. K. Dewhurst, I. Di Marco, C. Draxl, M. Dułak, O. Eriksson, J. A. Flores-Livas, K. F. Garrity, L. Genovese et al., Science 351, aad3000 (2016).

[16] C. G. Van de Walle and J. Neugebauer, J. Appl. Phys. (Melville, NY) 95, 3851 (2004).

[17] G. Makov and M. C. Payne, Phys. Rev. B 51, 4014 (1995).

[18] J. Neugebauer and M. Scheffler, Phys. Rev. B 46, 16067 (1992).

[19] M. D. Pashley, Phys. Rev. B 40, 10481 (1989).

[20] K. Shiraishi, J. Phys. Soc. Jpn. 59, 3455 (1990).

[21] M. Himmerlich, L. Lymperakis, R. Gutt, P. Lorenz, J. Neugebauer, and S. Krischok, Phys. Rev. B 88, 125304 (2013).

[22] S.-H. Yoo, M. Todorova, D. Wickramaratne, L. Weston, C. G. Van de Walle, and J. Neugebauer, npj Comput. Mater. (to be published).

[23] L. Lymperakis, P. H. Weidlich, H. Eisele, M. Schnedler, J.-P. Nys, B. Grandidier, D. Stiévenard, R. E. Dunin-Borkowski, J. Neugebauer, and P. Ebert, Appl. Phys. Lett. 103, 152101 (2013).

[24] G. Kresse and J. Furthmüller, Phys. Rev. B 54, 11169 (1996).

[25] G. Kresse and J. Furthmüller, Comput. Mater. Sci. 6, 15 (1996).

[26] P. E. Blöchl, Phys. Rev. B 50, 17953 (1994).

[27] J. P. Perdew, K. Burke, and M. Ernzerhof, Phys. Rev. Lett. 77, 3865 (1996).

[28] R. Elmér, M. Berg, L. Carlén, B. Jakobsson, B. Norén, A. Oskarsson, G. Ericsson, J. Julien, T. F. Thorsteinsen, M. Guttormsen, G. Løvhøiden, V. Bellini, E. Grosse, C. Müntz, P. Senger, and L. Westerberg, Phys. Rev. Lett. 78, 1396(E) (1997). 
[29] See Supplemental Material at http://link.aps.org/supplemental/ 10.1103/PhysRevMaterials.5.044605 for (i) the calculated bulk properties of $\mathrm{WZ} \mathrm{GaN,} \mathrm{AlN,} \mathrm{ZnO}$, and GaAs and comparisons with reports [38-48], (ii) the angle-dependent spontaneous polarization, (iii) the surface-bound charges and the cation interatomic distances at semipolar WZ surfaces, (iv) the electrostatic potential of WZ $\{1 \overline{1} 02\}$ slabs, and (v) electronic structures of WZ $\{1 \overline{1} 02\}$ and $\{1 \overline{1} 22\}$ slabs passivated by the conventional scheme and RIPS.

[30] R. D. King-Smith and D. Vanderbilt, Phys. Rev. B 47, 1651 (1993).

[31] J. E. Northrup, J. Neugebauer, R. M. Feenstra, and A. R. Smith, Phys. Rev. B 61, 9932 (2000).

[32] C. Adelmann, J. Brault, G. Mula, B. Daudin, L. Lymperakis, and J. Neugebauer, Phys. Rev. B 67, 165419 (2003).

[33] M. S. Miao, A. Janotti, and C. G. Van de Walle, Phys. Rev. B 80, 155319 (2009).

[34] J. Neugebauer, T. Zywietz, M. Scheffler, J. E. Northrup, and C. G. Van de Walle, Phys. Rev. Lett. 80, 3097 (1998).

[35] L. Lymperakis, J. Neugebauer, M. Albrecht, T. Remmele, and H. P. Strunk, Phys. Rev. Lett. 93, 196401 (2004).

[36] H. Lüth, in Solid Surfaces, Interfaces and Thin Films (Springer, Berlin, 2010), pp. 323-376.
[37] R. Vetury, Polarization induced 2DEG in AlGaN/GaN HEMTs: On the origin, DC and transient characterization Ph.D. thesis, University of California, Santa Barbara, 2000.

[38] C. Stampfl and C. G. Van de Walle, Phys. Rev. B 59, 5521 (1999).

[39] I. Vurgaftman and J. R. Meyer, J. Appl. Phys. (Melville, NY) 94, 3675 (2003).

[40] H. Schulz and K. Thiemann, Solid State Commun. 23, 815 (1977).

[41] B. Meyer and D. Marx, Phys. Rev. B 67, 035403 (2003).

[42] S. C. Abrahams and J. L. Bernstein, Acta Crystallogr. Sect. B: Struct. Crystallogr. Cryst. Chem. 25, 1233 (1969).

[43] D. C. Reynolds, D. C. Look, B. Jogai, C. W. Litton, G. Cantwell, and W. C. Harsch, Phys. Rev. B 60, 2340 (1999).

[44] T. Cheiwchanchamnangij and W. R. L. Lambrech, Phys. Rev. B 84, 035203 (2011)

[45] F. Bernardini, V. Fiorentini, and D. Vanderbilt, Phys. Rev. B 56, R10024(R) (1997).

[46] F. Bernardini, V. Fiorentini, and D. Vanderbilt, Phys. Rev. B 63 193201 (2001).

[47] C. E. Dreyer, A. Janotti, C. G. Van de Walle, and D. Vanderbilt, Phys. Rev. X 6, 021038 (2016).

[48] A. Dal Corso, M. Posternak, R. Resta, and A. Baldereschi, Phys. Rev. B 50, 10715 (1994). 